\title{
SPECTRAL ENERGY DISTRIBUTIONS OF NUCLEI OF PECULIAR GALAXIES
}

\author{
J. B. OKE \\ Hale Observatories, Pasadena, California, U.S.A.
}

\section{Introduction}

Studies of galaxies during the last seventy years have turned up a large array of peculiar objects. Vigorous work on these was rare until a few years ago when quasars were discovered. It then became clear that an understanding of quasars might come from an understanding of the less-extreme peculiar galaxies. It was further clear that even the physical processes occurring in nuclei of peculiar galaxies were far from easy to understand.

There are several groups of objects with which I will be concerned in this paper:

(1) Seyfert Galaxies. NGC 1068 was recognized as peculiar by Fath (1908) in 1908. The first studies of NGC 4151 were made in 1918 by Campbell and Moore (1918) at the Lick Observatory. Seyfert (1943) made the first quantitative study of a substantial number of galaxies with small bright nuclei. These 'Seyfert' galaxies are nearly all very close objects, and, with the possible exception of NGC 1275, are spiral galaxies. They all have tiny, bright nuclei.

(2) Vorontsov-Velyaminov and Krasnogorskaya (1962) and Vorontsov-Velyaminov and Arhipova $(1963,1964,1968)$ have published a catalog of peculiar galaxies. Many of these turn up in other lists such as those of Markarian (see below).

(3) Haro (1956) also has published a list of peculiar galaxies, of which a few have been studied.

(4) Zwicky Compact Galaxies. Since 1964 Zwicky has distributed seven lists of galaxies which have high surface brightness per square sec of arc. He also includes parts of galaxies with this same property. They are all nonstellar on the 48 -in. Schmidt survey and, therefore, should be extragalactic objects. Their spectra have been studied mainly by Zwicky $(1964,1966,1967)$ and Sargent (1970b). Many of these galaxies are very distant.

(5) Markarian Galaxies. Markarian has published three lists of peculiar galaxies (Markarian 1967, 1969a, 1969b). They have been discovered from objective prism spectra and are characterized by strong ultraviolet radiation as compared with normal galaxies. These objects are relatively close. Spectra have been studied by Weedman and Khachikian (1968, 1969), Arakelian, Dibai, and Yesipov (1970), and Sargent (1970a).

(6) Radio Galaxies. Many galaxies identified with radio sources are not peculiar in appearance. Other radio galaxies, usually referred to as $\mathrm{N}$ galaxies, (Matthews et al., 
1964) are peculiar and have tiny, very bright nuclei. Spectra have been studied mainly by Schmidt (1965), Sandage (1966), and M. Burbidge (1967).

(7) Quasars. In this paper quasars are discussed in terms of their similarities to the nuclei of peculiar galaxies.

\section{Observational Techniques}

In the visual spectral range at least three kinds of observations can be made:

(1) Direct Photographs. These can be used to classify the objects to determine, for instance, the relationship of the bright nucleus to the background galaxy. This type of observation has already been discussed in this Symposium by Dr Morgan.

(2) Slit Spectra. These indicate for the nuclear region what lines are present, what the line shapes are, what velocity fields exist, and the red shift. Analysis of line ratios may help to give the electron density $N_{e}$ and electron temperature $T_{e}$.

(3) Photoelectric Spectrometer Observations. These give absolute line intensities over the whole spectral range, and line intensities in the far red and near infrared which is not usually accessible with slit spectrographs. The technique is also the best method for finding very broad, low-contrast emission and absorption features. Finally, the absolute spectral energy distribution of the continuous spectrum can be obtained. From this one can hope to separate the radiation from stars in the galactic nucleus from the other contributors to the radiation.

(4) Infrared Photometry. These measurements can be put on an absolute basis and extend by a large factor the wavelength range covered by normal spectrophotometry mentioned in (3) above. Dr Neugebauer will discuss these kinds of observations in a later paper.

In this paper I will discuss, almost exclusively, data obtained with photoelectric spectrometers.

\section{Spectral Properties}

Peculiar galaxies of the sort described above have a wide range of properties insofar as their spectra are concerned. Many have very strong emission lines. Others may show only $\mathrm{H} \alpha$ and $\lambda 3727$ in emission, while occasionally no emission lines are seen at all. In some objects all the emission lines are narrow and similar in breadth. Others have the 'Seyfert characteristic', namely very broad permitted lines and narrower forbidden lines. Most objects discussed in this paper have this latter characteristic. In a few compact galaxies the forbidden lines are weak or absent; usually in these objects emission lines of permitted FeII are observed, indicating densities of $10^{6}$ or $10^{7}$ electrons $\mathrm{cm}^{-3}$ or more.

The continuous spectra of the reddest objects are very similar to those of normal galaxies; broad absorption features caused by the $\mathrm{MgI}_{\mathrm{I}}$ lines, the G-band and the $\mathrm{H}$ and $\mathrm{K}$ lines of $\mathrm{Ca} I \mathrm{I}$ are usually present. The bluer objects have smooth continuous spectra with little or no evidence of absorption features. In these objects the ultraviolet radiation may be abnormally high. 


\section{Sources of Continuous Radiation}

(1) The stars in the background galaxy will contribute to the total observed radiation. It will be assumed that the absolute energy distribution of any stellar contribution is similar to that observed in the nucleus of M 31 (Oke and Sandage, 1968). This may not be a valid assumption since Wampler (1971) has shown that the stellar radiation near the nucleus of NGC 1068 is significantly bluer than that from the nucleus of M31.

(2) Bound-free and free-free radiation from hydrogen. This must be present since Balmer emission lines are often very strong. If this contribution is large, a Balmer jump in emission may be observed as in NGC 4151 (Oke and Sargent, 1968). Twophoton emission could occur although most estimates of the electron density in these objects rule it out.

(3) Non-thermal radiation, such as synchrotron and inverse Compton radiation may be present. It is assumed that any nonthermal contributor has a spectrum of the form $F_{v} \propto v^{\alpha}$ where $v$ is the frequency and $\alpha$ the spectral index.

(4) Large amounts of hot interstellar dust could radiate substantially in the infrared. In such a case, any variability of this component must have time scales of many years.

All of the above radiation sources may be affected by interstellar reddening. Corrections for insterstellar reddening in our own Galaxy are usually easily made, since most peculiar galaxies which have been studied are at high galactic latitudes. Interstellar reddening within the central region of the observed galaxy is much harder to estimate. Wampler (1968b) has studied the ratio of infrared to blue sulphur forbidden lines in Seyfert galaxies and finds strong evidence for reddening. A comparison of Paschen and Balmer line intensities and, in principle at least, the Balmer decrement, can also be used to determine the amount of reddening.

\section{Distance Scale Problems and Selection Effects}

Ideally, one would like to always observe only the bright nucleus of a peculiar galaxy out to some limiting distance, say 25 or 50 parsec. This can never be done in practice because (a) one always observes along a line-of-sight which penetrates the whole galaxy, and (b) galaxies are at different distances and only in a few cases is a resolution of 50 parsec possible. The classical 'Seyfert' galaxies are all close and it is possible to isolate the tiny, bright nucleus quite well. But even in NGC 4151, Wampler (1971) has shown that the stellar contribution within a few sec of arc of the center is appreciable and variable with wavelength. More information can be obtained about the stellar background by measuring radiation through apertures of different sizes, but even in this circumstance some model for the light distribution is needed to predict the contribution at the center.

In the case of a distant Zwicky compact galaxy, the observing aperture may include not only light from the stellar nucleus, but also light from most of the background 
galaxy as well. Such a galaxy is discovered only because the nucleus is exceedingly bright compared with the whole galaxy. A galaxy such as NGC 4151, which has only a moderately bright nucleus, would probably never be detected as a peculiar galaxy if it were at a large distance. As a result, galaxies with only mild activity in the nucleus are all nearby objects. Those objects which have violent activity are seen only at very large distances, presumably because they are very rare.

Because the amount of background stellar radiation depends so much on distance, it is necessary to separate this component of the radiation from the other contributors. Only if this can be done will it be possible to study adequately the conditions in the nuclei of peculiar galaxies.

\section{Observational Material}

Data will be presented which are representative of spectrophotometric observational results for 12 Markarian galaxies, 14 Zwicky compact objects, and 16 radio galaxies.

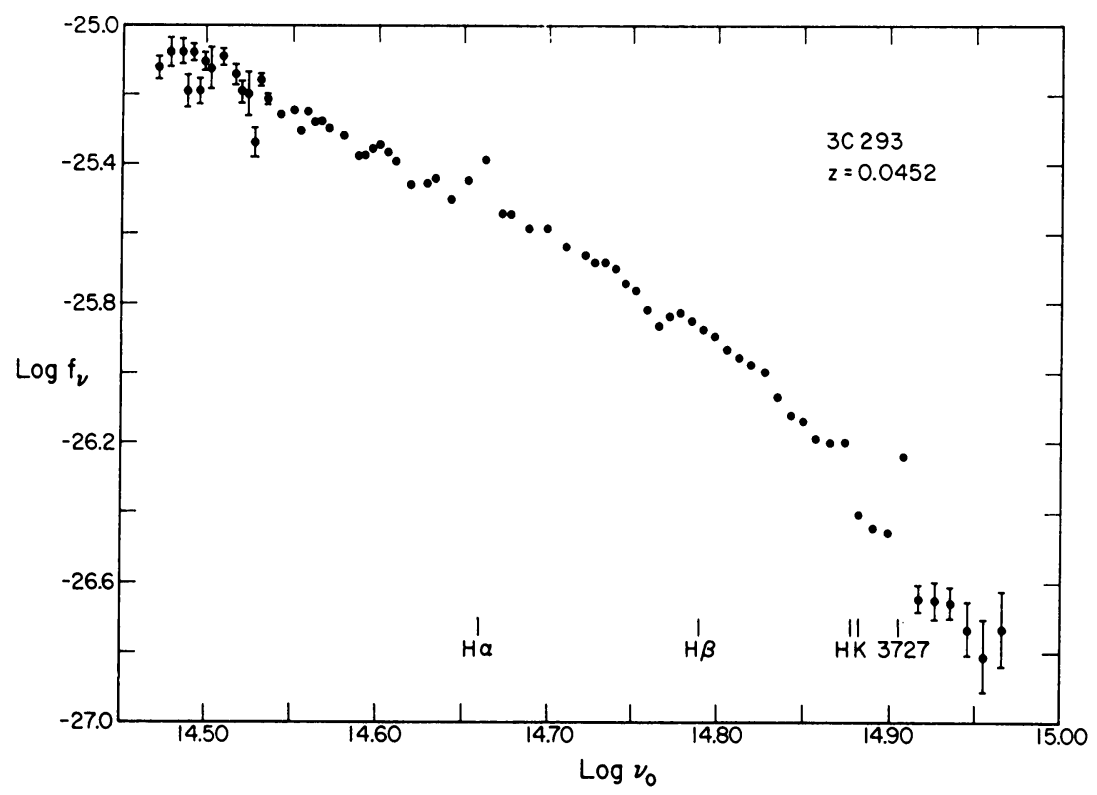

Fig. 1. The absolute spectral energy distribution of the radio galaxy 3C 293. The logarithm of the apparent flux $f_{v} \mathrm{erg} \mathrm{s}^{-1} \mathrm{~cm}^{-2} \mathrm{~Hz}^{-1}$ is plotted against the rest frequency $v_{0}$. Positions of several emission lines and the $\mathrm{H}$ and $\mathrm{K}$ lines of CaII are marked. Standard deviations are shown if they are greater than 0.02 in $\log f_{v}$. The red shift is indicated.

The results are shown and described in Figures 1 to 7 . The object 3C 293 is a radio galaxy of normal appearance. The spectral energy distribution is almost identical with that of ordinary elliptical galaxies except for the presence of $\mathrm{H} \alpha$ and $\lambda 3727$ of [O $\mathrm{II}]$ in emission. The spectral index, $\alpha$, which will be defined over the spectral range 


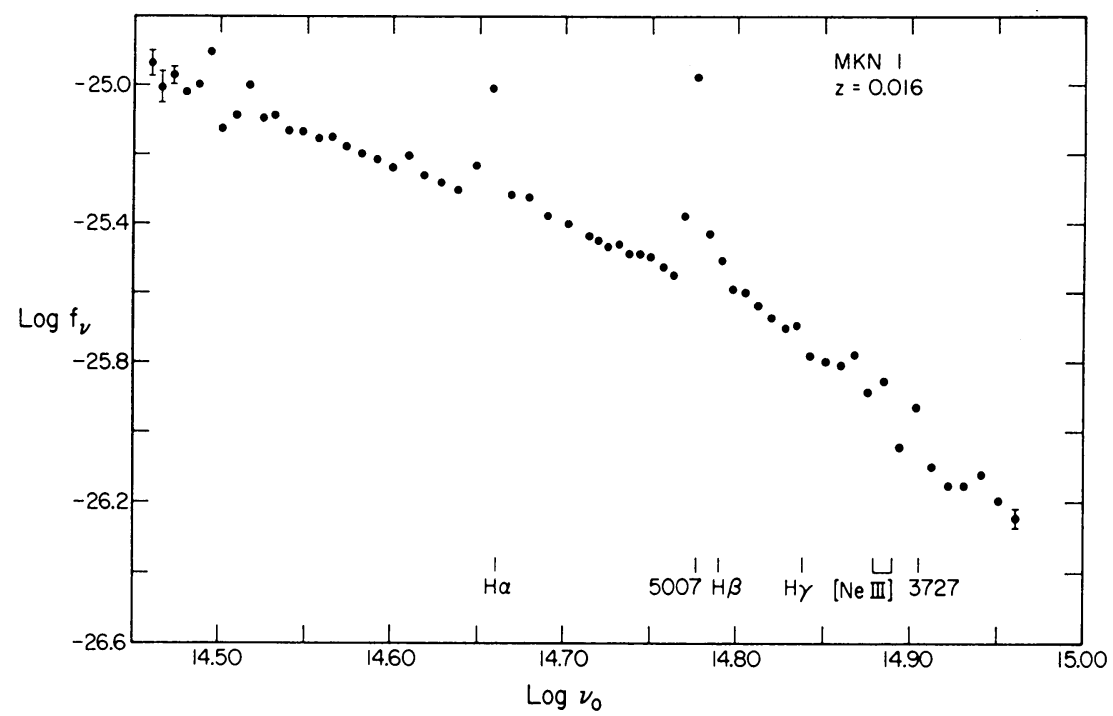

Fig. 2. Markarian No. 1.

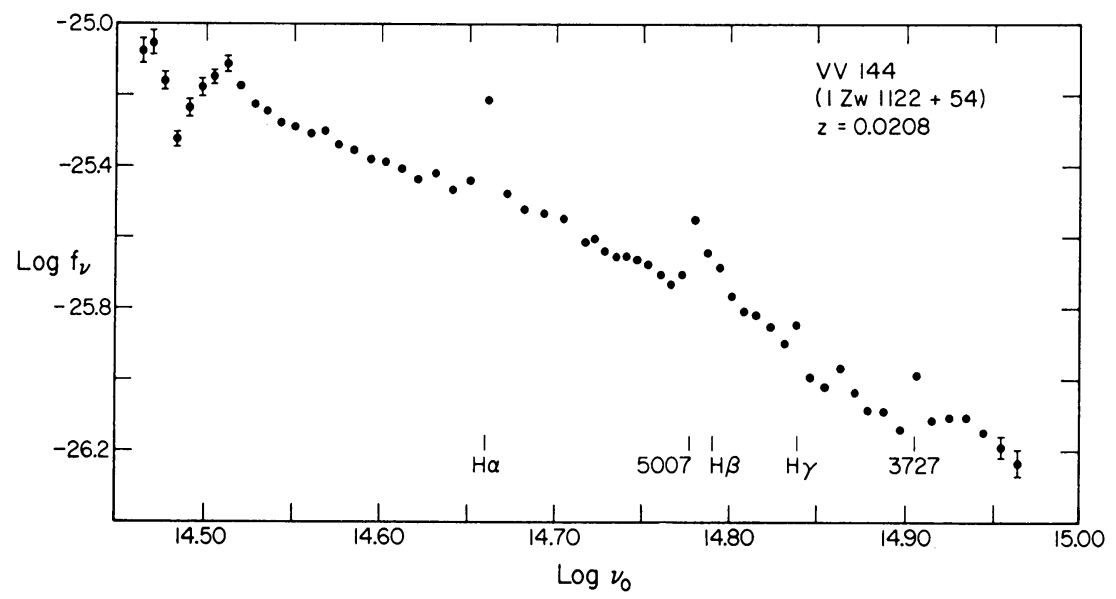

Fig. 3. Vorontsov-Velyaminov object VV 144.

from $4000 \AA$ to $10000 \AA$ is -2.7 and typical of the central regions of normal galaxies.

Markarian No. 1 (which is also NGC 449) and the Vorontsov-Velyaminov object VV 144 (also the $Z$ wicky compact I $Z_{w} 1122+54$ ) have energy distributions which are mixtures of a background galaxy and some non-thermal source. The spectral indices are both near -2.0 and there is strong evidence that the $\mathrm{H}$ and $\mathrm{K}$ lines of CaII are present in absorption.

The radio galaxy $3 C 382$ also has a spectral index of -2.0 from $4000 \AA$ to $10000 \AA$, 


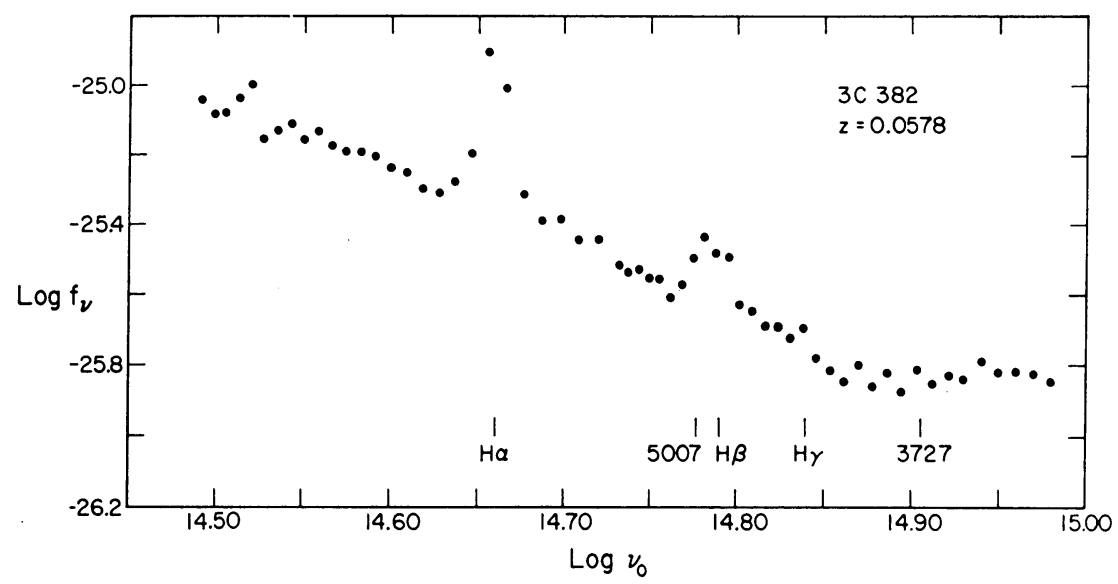

Fig. 4. Radio Galaxy 3C 382.

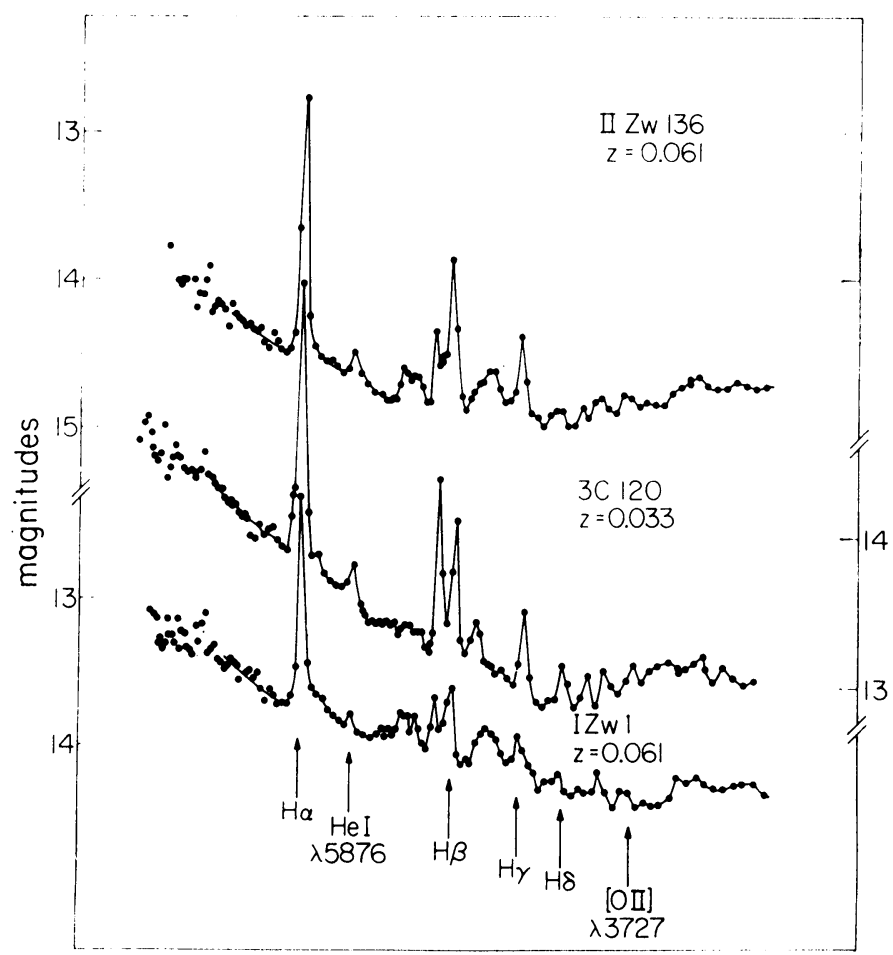

Fig. 5. Three objects IIZw 136 (IIZw $2130+09$ ), 3C 120, and IZw 1 (IZw $0051+12$ ). The vertical scale is $-2.5 \log f_{v}+$ const. and the horizontal scale is $1 / \lambda$ where $\lambda$ is the observed wavelength in microns. The emission on either side of $\mathrm{H} \beta$ and $\lambda 5007$ of [OIII] is due to FelI. 


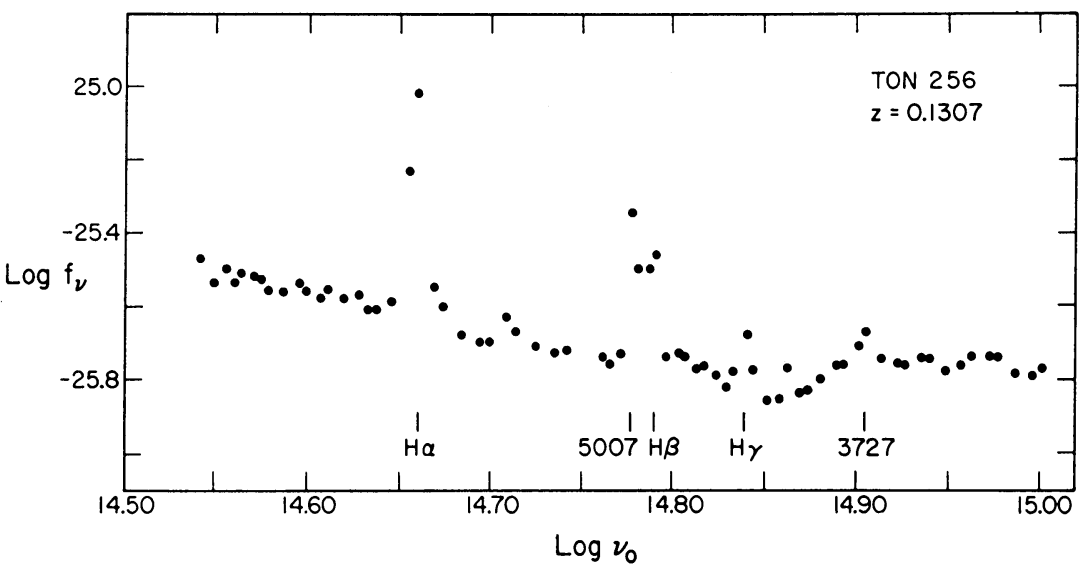

Fig. 6. Tonanzintla object Ton 256. Axes are the same as in Figure 1. The permitted lines are very broad.

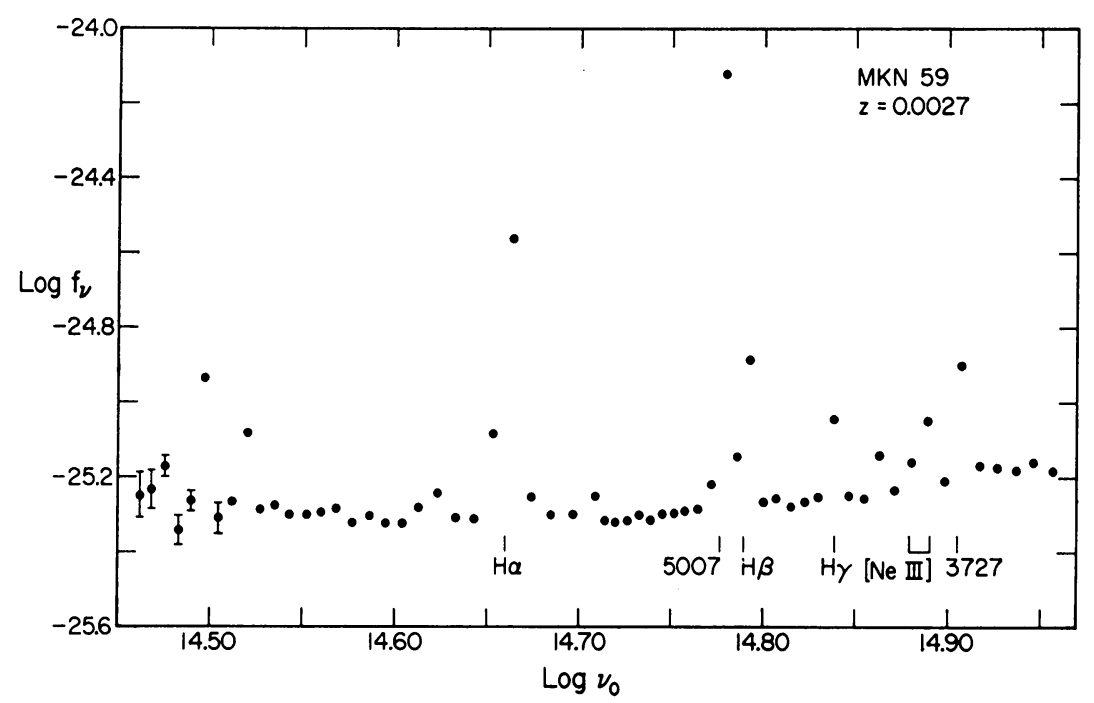

Fig. 7. Markarian No. 59. The emission lines are very strong and sharp. The object is probably a very large Hil region.

but in this object the spectrum becomes flat below $4000 \AA$. This could be caused by a Balmer jump in emission or by many unresolved emission lines in this spectral range. Many quasi-stellar sources have energy distributions similar to that in this object. Schmidt (1965) has obtained a spectrum of this object and finds no evidence for absorption lines.

The three objects II Zw $2130+09,3 \mathrm{C} 120$, and I Zw $0051+12$ have been observed by Sargent and Oke and have spectral indices of $-1.13,-1.93$, and -1.24 respec- 
tively. Both Zwicky objects have strong FeII lines (Sargent, 1968a, 1968b) and weak or absent forbidden lines. It is not certain whether the N-galaxy 3C 120 has Fe II lines. As in 3C 382, all the spectra are flat in the ultraviolet and there is no evidence for absorption features. Ton 256 has a spectral energy distribution very much like those of the two Zwicky objects mentioned above.

Markarian No. 59 is one of only three objects so far observed in which the spectral index is positive, being +0.2 . The forbidden and permitted lines are very strong and sharp. It is suspected that this object is a very large HII region.

Absolute spectral energy distributions similar to those described above for peculiar galaxies have also been obtained for quasi-stellar sources by Oke $(1966,1967 \mathrm{a})$ Wampler (1967a, 1967b, 1968a), and by Oke et al. (1970). A comparison of energy distributions of quasi-stellar sources with those of galaxies in which absorption lines are not seen, shows some striking similarities.

(a) The range of spectral indices is similar.

(b) Many galaxies, such as those mentioned above and quasars such as 3C 345, PKS 0405-12, and 3C 334, have spectral energy distributions which become flat below a rest wavelength of $4000 \AA$.

(c) Some quasi-stellar sources, such as 3C 323.1, have energy distributions, line strengths, and line breadths which are almost identical with those of peculiar galaxies such as the Braccesi object B 264 and Ton 256.

\section{General Properties of Spectral Energy Distributions of Peculiar Galaxies and Quasars}

It has been shown by Sandage, for example, (Sandage, 1967b) that there is a regular progression of colors as one goes from quasi-stellar sources, through $\mathrm{N}$-galaxies to normal elliptical galaxies. This progression is, however, somewhat masked in the broad-band color measurements by the strong emission lines often present in the spectrum. It is, therefore, preferable to use the spectral index $\alpha$ of the continuous energy distribution as a measure of color. As indicated above, $\alpha$ is here defined between $4000 \AA$ and $10000 \AA$ and the region below $4000 \AA$ is neglected.

Another quantity which is relevant for these objects is the absolute flux $F_{v_{0}} \mathrm{erg} \mathrm{s}^{-1}$ $\mathrm{Hz}^{-1}$ of the continuum at a fixed rest frequency $v_{0} ; v_{0}$ is chosen to correspond to $4000 \AA$. Distances are calculated using a Hubble constant of $100 \mathrm{~km} \mathrm{~s}^{-1} \mathrm{Mpc}^{-1}$ and a cosmological constant $q_{0}=+1$. The results for the sample of objects being discussed in this paper are shown in Figure 8 where the spectral index $\alpha$ is plotted against $\log F_{v_{0}}$. A few specific objects are marked. Quasars, assumed to be at cosmological distances, are shown by crosses. If they are not at cosmological distances, they shift horizontally far to the left. $G$ marks the location of the brightest cluster galaxy when measured out to an isophote corresponding to $25 \mathrm{mag}$. $\operatorname{arc~sec}^{-2}$.

The family of curves shows the result of combining the spectral energy distribution of a bright galaxy with various contributions of a power-law spectrum. The bright galaxy is assumed to be somewhat fainter than the brightest cluster galaxy, since it 


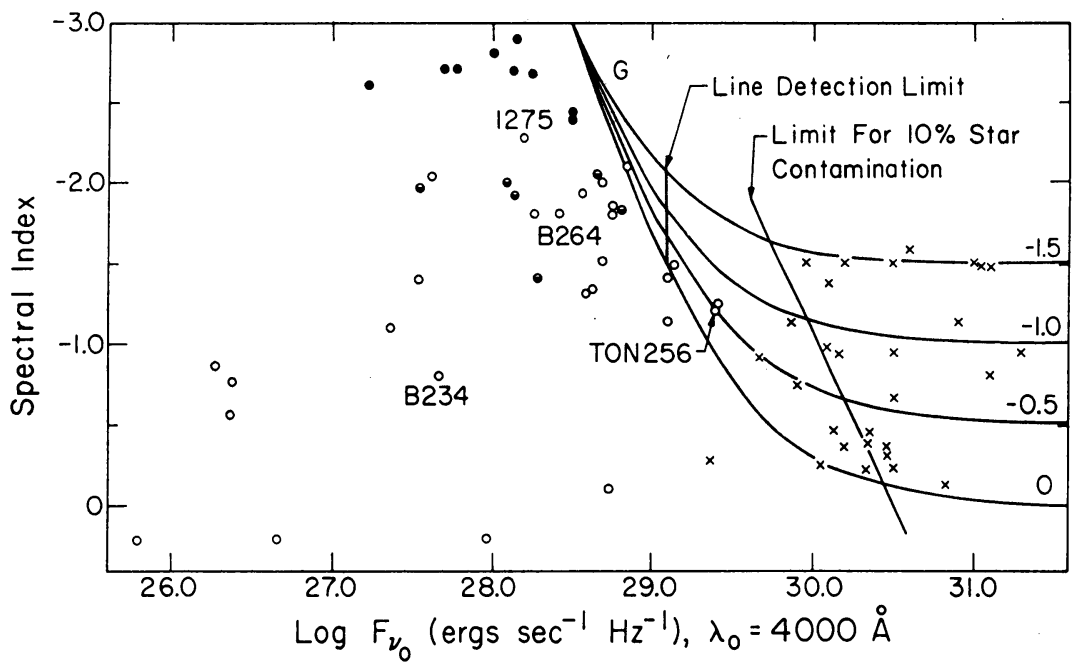

Fig. 8. The spectral index $\alpha$ defined over the range from $4000 \AA$ to $10000 \AA$ plotted against the absolute flux at a rest wavelength of $4000 \AA$. Quasars are plotted as crosses. $G$ is the location of the brightest cluster galaxy.

is usually measured with an aperture which does not include all the light. Each curve goes from a pure galaxy spectrum to a pure power-law spectrum. The oblique straight line shows the limit beyond which radiation from even a very bright galaxy will not contribute more than $10 \%$ to the total radiation at any wavelength. It is clear that most, but not quite all, quasi-stellar sources have energy distributions which are virtually unaffected by any background galaxy.

Another limit which can be placed in this figure is the point beyond which the $\mathrm{H}$ and $\mathrm{K}$ lines of $\mathrm{Ca}$ II would not be detected. Because observational data are still not very precise, and there are often emission lines near $\mathrm{H}$ and $\mathrm{K}$, it is estimated that the radiation at $\mathrm{H}$ and $\mathrm{K}$ can be diluted by approximately a factor 3 and still leave the lines detectable. This limit is shown by the vertical line in Figure 8; to its right, the $\mathrm{H}$ and $\mathrm{K}$ lines would probably not be detected using data of typical accuracy at the present time. This same vertical line also represents the limit of detectability of the $\mathrm{MgI}$ lines at 25180 . This feature is weaker than $\mathrm{H}$ and $\mathrm{K}$, but is in a more favorable spectral region for detection.

The family of curves shown in Figure 8 applies only for a contributing galaxy of fixed luminosity. If the galaxy is made fainter, the curves all shift horizontally to the left. When allowance is made for this, the following general conclusions can be reached:

(1) For $\alpha<-2.1$, the $\mathrm{H}$ and $\mathrm{K}$ lines should always be visible in the observed spectral energy distribution.

(2) For $-2.1<\alpha<-1.4$, the $\mathrm{H}$ and $\mathrm{K}$ lines may or may not be seen, depending on the galaxy luminosity and the spectral index of the non-thermal source.

(3) For $\alpha>-1.4$, the $H$ and $K$ lines should not be seen. 
It should be emphasized that the above remarks assume a modest accuracy for the observations. As observational accuracy and wavelength resolution improve, the limits will shift. It also should be pointed out that it is possible to make detailed composite energy distributions. When these are compared with observations, somewhat more definite conclusions can be reached concerning the relative importance of nonthermal and galaxy radiation.

In Figure 8, the solid dots denote galaxies where $\mathrm{H}$ and $\mathrm{K}$ lines are seen. The half-filled circles denote those galaxies where $\mathrm{H}$ and $\mathrm{K}$ may be present. $\mathrm{H}$ and $\mathrm{K}$ are not seen in those objects shown by open circles. With the exception of NGC 1275, for which better observations are needed, the various domains defined above are filled with the expected kinds of objects.

The three objects with positive spectral indices are all like Mkn 59 which was shown in Figure 7. They are all probably Hil regions. The three objects, B 234, B 264, and Ton 256 which have often, in the past, been called quasi-stellar objects, have been shown by Arp (1970b) to be galaxies.

\section{Variability}

Another tool for separating the stellar and non-thermal radiation is available when the object is variable, since it is reasonable to assume that any light variability is associated only with the non-thermal radiation. If it could be assumed that the nonthermal spectral index is independent of brightness, then the separation of the nonthermal and galaxy contribution would be trivial. In practice, this assumption is almost certainly incorrect and the whole problem becomes more complicated, and other data such as absorption line strengths are desirable.

A good example of a variable object which has been studied in some detail is 3C 371 (Oke, 1967b; Sandage, 1967a). When the object is faint, features such as the H and K lines of $\mathrm{CaII}$ and the $\lambda 5180$ band of $\mathrm{MgI}$ are just detectable. When the object is bright, these are not seen. Furthermore, the continuous energy distribution has a different slope depending on the brightness, as is expected of a composite spectrum when the two components are quite different. A reasonable separation of the galaxy and nonthermal contributions can be made. Arp (1970a) has shown that this object is a large galaxy with the outer regions being very faint.

Much more work should be done to find variable galaxies and study their spectral changes.

\section{Conclusions}

All of the objects which have been discussed in this paper, apart from quasars, are extended and must be galaxies. Since the galaxy is visible, it is clear that the observed spectral energy distribution must have some contribution from stars in addition to the non-thermal component. It is important to separate these components where possible in order (a) to study the non-thermal radiation (b) to investigate the nature of the stellar component in the nucleus, and (c) to determine how much energy is 
coming from each source of radiation and consequently to learn what kinds of galaxies produce what kinds of non-thermal radiation.

I have attempted to show that progress can and is being made in these directions. It is clear that more objects should be observed spectrophotometrically. In particular, emphasis should be placed on variable galaxies. It will probably be useful to study objects by measuring colors and magnitudes through various apertures. Finally, spectrophotometric data should be improved by increasing the accuracy of the observations and also by increasing to some extent the wavelength resolution. If these improvements are made, it will be possible to detect absorption features such as the $\mathrm{H}$ and $\mathrm{K}$ lines, the G-band and the $\mathrm{MgI}$ band in many more peculiar galaxies.

\section{Acknowledgement}

The author wishes to thank Mr. B. Turnrose who helped to reduce and analyse much of the data presented in this paper. This work was supported in part through grant No. NGR 05-002-134 from the National Aeronautics and Space Administration.

\section{References}

Arakelian, M. A., Dibai, E. A., and Yesipov, V. F.: 1970, Astrofizika 6, 39.

Arp, H. C.: 1970a, Astrophys. Letters 5, 75.

Arp, H. C.: 1970b, Astrophys. J. $162,811$.

Burbidge, E. M.: 1967, Astrophys. J. Letters 149, L51.

Campbell, W. W. and Moore, J. H.: 1918, Pub. Lick Obs. 13, 122.

Fath, E. A.: 1908, Lick. Obs. Bull. 5, 71.

Haro, G.: 1956, Bol. Obs. Tonantzintla Tacubaya $2,8$.

Markarian, B. E.: 1967, Astrofizika 3, 55.

Markarian, B. E.: 1969a, Astrofizika 5, 443.

Markarian, B. E.: 1969b, Astrofizika 5, 581.

Matthews, T. A., Morgan, W. W., and Schmidt, M.: 1964, Astrophys. J. 140, 35.

Oke, J. B.: 1966, Astrophys. J. 145, 668.

Oke, J. B.: 1967a, Astrophys. J. 147, 901.

Oke, J. B.: 1967b, Astrophys. J. Letters 150, L5.

Oke, J. B., Neugebauer, G., and Becklin, E. E.: 1970, Astrophys. J. 159, 341.

Oke, J. B. and Sandage, A.: 1968, Astrophys. J. 154, 21.

Oke, J. B. and Sargent, W. L. W.: 1968, Astrophys. J. 151, 807.

Sandage, A.: 1966, Astrophys. J. 145, 1.

Sandage, A.: 1967a, Astrophys. L. Letters 150, L9.

Sandage, A.: 1967b, Astrophys. J. Letters 150, L177.

Sargent, W. L. W.: 1968a, Astron. J. 73, 893.

Sargent, W. L. W.: 1968b, Astrophys. J. Letters 151, L31.

Sargent, W. L. W.: 1970a, Astrophys. J. 159, 765.

Sargent, W. L. W.: 1970b, Astrophys. J. 160, 405.

Schmidt, M.: 1965, Astrophys. J. 141, 1.

Seyfert, C. K.: 1943, Astrophys. J. 97, 28.

Vorontsov-Velyaminov, B. A. and Arhipova, V.: 1963, Trudy Gos. Astron. Inst. Sternberga 33. Vorontsov-Velyaminov, B. A. and Arhipova, V.: 1964, Trudy Gos. Astron. Inst. Sternberga 34. Vorontsov-Velyaminov, B. A. and Arhipova, V.: 1968, Trudy Gos. Astron. Inst. Sternberga 38. Vorontsov-Velyaminov, B. A. and Krasnogorskaya, A.: 1962, Trudy Gos. Astron. Inst. Sternberga 32. Wampler, E. J.: 1967a, Astrophys. J. 147, 1.

Wampler, E. J.: 1967b, Astrophys. J. Letters 148, L101. 
Wampler, E. J.: 1968a, Astrophys. J. 153, 19.

Wampler, E. J.: 1968b, Astrophys. J. Letters 154, L53.

Wampler, E. J.: 1971, Astrophys. J. 164, 1.

Weedman, D. W. and Khachikian, E. Ye.: 1968, Astrofizika 4, 587.

Weedman, D. W. and Khachikian, E. Ye.: 1969, Astrofizika 5, 113.

Zwicky, F.: 1964, Astrophys. J. 140, 1467.

Zwicky, F.: 1966, Astrophys. J. 143, 192.

Zwicky, F.: 1967, Adv. Astron. Astrophys. 5, 267.

\section{Discussion}

Wray: I would like to draw attention to the fact that the strength of $N_{1}$ and $N_{2}$ exceeds that of $\mathrm{H} \beta$ in some of the nuclear regions and inner HII regions in ordinary galaxies, as shown by Dr. Rubin as well as generally in HII regions in the outer parts of ordinary spiral galaxies, as observed by Searle. Perhaps it is possible that the relative strength of $N_{1}, N_{2}$ compared with the inner core of the $\mathrm{H} \beta$ line (neglecting the broad wings) occurring in a number of Seyfert galaxies and compact galaxies is due in some degree to physical processes similar to those occurring in nuclear regions and inner $\mathrm{HII}$ regions of some ordinary galaxies, a likely mechanism being excitation by a component of $O B$ stars. It is interesting to note that these sharp hydrogen line cores are absent from spectra of a number of quasi-stellar objects and that a number of these objects, as you have shown, most probably do not contain significant, if any, underlying stellar populations. This suggests that the broad wings of the hydrogen lines in Seyfert galaxies also are not likely to be due to stellar excitation. In short it appears that two excitation mechanisms may be operating; OB stars for objects ranging from ordinary galactic Hil regions to at least a part of the Seyfert galaxy and compact galaxy emission radiation, and a second source responsible for broad emission lines in objects ranging from Seyfert galaxies and some compact galaxies through quasi-stellar objects. 